\title{
Oligofructose and inulin modulate glucose and amino acid metabolism through propionate production in normal-weight and obese cats
}

\author{
Adronie Verbrugghe ${ }^{1 *}$, Myriam Hesta $^{1}$, Kris Gommeren ${ }^{2}$, Sylvie Daminet ${ }^{2}$, Birgitte Wuyts ${ }^{3}$, Johan Buyse ${ }^{4}$ \\ and Geert P. J. Janssens ${ }^{1}$ \\ ${ }^{1}$ Laboratory of Animal Nutrition, Faculty of Veterinary Medicine, Ghent University, Heidestraat 19, B-9820 Merelbeke, Belgium \\ ${ }^{2}$ Department of Small Animal Medicine, Faculty of Veterinary Medicine, Ghent University, Salisburylaan 133, B-9820 Merelbeke, \\ Belgium \\ ${ }^{3}$ Department of Clinical Chemistry, Laboratory of Metabolic Disorders, University Hospital Ghent, De Pintelaan 185, B-9000 \\ Ghent, Belgium \\ ${ }^{4}$ Department of Biosystems, Laboratory of Livestock Physiology, Immunology and Genetics, K.U. Leuven, Kasteelpark Arenberg 30, \\ B-3001 Leuven, Belgium \\ (Received 21 July 2008 - Revised 5 January 2009 - Accepted 27 January 2009 - First published online 9 March 2009)
}

\begin{abstract}
The effect of dietary oligofructose and inulin supplementation on glucose metabolism in obese and non-obese cats was assessed. Two diets were tested in a crossover design; a control diet high in protein ( $46 \%$ on DM basis), moderate in fat (15\%), low in carbohydrates ( $27 \%)$, but no soluble fibres added; and a prebiotic diet, with $2.5 \%$ of a mixture of oligofructose and inulin added to the control diet. Eight non-obese and eight obese cats were allotted to each of two diets in random order at intervals of 4 weeks. At the end of each testing period, intravenous glucose tolerance tests were performed. Area under the glucose curve $\left(\mathrm{AUC}_{\mathrm{gluc}}\right)$ was increased $(P=0.022)$ and the second insulin peak was delayed $(P=0 \cdot 009)$ in obese compared to non-obese cats. Diets did not affect fasting plasma glucose concentrations, blood glucose response at each glucose time-point after glucose administration, $\mathrm{AUC}_{\text {gluc }}$, fasting serum insulin concentrations, area under the insulin curve, and height and appearance time of insulin response. Yet, analysis of acylcarnitines revealed higher propionylcarnitine concentrations $(P=0.03)$ when fed the prebiotic diet, suggesting colonic fermentation and propionate absorption. Prebiotic supplementation reduced methylmalonylcarnitine $(P=0.072)$ and aspartate aminotransferase concentrations $(P=0.025)$, both indicating reduced gluconeogenesis from amino acids. This trial evidenced impaired glucose tolerance and altered insulin response to glucose administration in obese compared to non-obese cats, regardless of dietary intervention; yet modulation of glucose metabolism by enhancing gluconeogenesis from propionate and inhibition of amino acid catabolism can be suggested.
\end{abstract}

Carbohydrate metabolism: Cats: Dietary fibre: Obesity

Diabetes mellitus has become one of the major endocrine disorders in cats. Recently a tendency towards a higher incidence of this disease was reported by Prahl et al. ${ }^{(1)}$ and might be due to a rise in the frequency of the major predisposing factors such as obesity and physical inactivity ${ }^{(2)}$. However, the current dietary treatments for feline diabetes mellitus, especially the use of soluble fibres, originates from extrapolation of the results from human and canine studies. To our knowledge, the effect of soluble fibres on carbohydrate metabolism in both healthy and insulin-resistant cats (obese or diabetic cats) has not been studied.

Soluble fibres such as oligofructose and inulin have been shown to modulate glycaemia and insulinaemia, although effects may depend on nutritional (fasting $v$. postprandial) and pathological (diabetes mellitus, obesity) conditions ${ }^{(3)}$.
In the literature, two hypotheses are proposed. At first, soluble fibres might impair digestion of macronutrients by delaying gastric emptying and/or by reducing small intestinal transit time ${ }^{(4-6)}$. Secondly, the production of SCFA in the hindgut is stimulated by offering soluble fibres as energy source for colonic microbial flora. After being absorbed, the SCFA, especially propionate, might modify the hepatic glucose metabolism by reducing hepatic gluconeogenesis and/or enhancing glycolysis; consequently, blood glucose concentrations will be decreased ${ }^{(3)}$. Hepatic gluconeogenesis might also be influenced indirectly by lowering the plasma fatty acid concentration, since increased plasma fatty acid availability may induce impaired insulin sensitivity by promoting fatty acid oxidation and inhibiting glucose uptake and oxidation but stimulating hepatic glucose $\operatorname{production}^{(7)}$.

\footnotetext{
Abbreviations: ALT, alanine aminotransferase; AST, aspartate aminotransferase; $\mathrm{AUC}_{\text {gluc }}$, area under the glucose curve; $\mathrm{AUC}_{\mathrm{ins}}$, area under the insulin curve; BCS, body condition score; C-diet, control diet; glucose $t_{1 / 2}$, half-life for glucose disappearance; IVGTT, intravenous glucose tolerance test; HOMA, homeostasis model assessment; $K_{\text {gluc }}$, glucose disappearance coefficient; P-diet, prebiotic diet; QUICKI, quantitative insulin sensitivity check index; $\mathrm{T}_{3}$, tri-iodothyronine; $\mathrm{T}_{4}$, thyroxine.

* Corresponding author: Dr Adronie Verbrugghe, fax +32 926478 48, email Adronie.Verbrugghe@UGent.be
} 
In other less strict carnivores than cats, such as dogs, few studies investigating the effect of soluble dietary fibre have been performed, resulting in a decreased postprandial glycaemia and/or insulinaemia ${ }^{(8-12)}$. Yet, to date, no data are available on the effect of prebiotics on the carbohydrate metabolism in more strict carnivorous species such as the cat.

Not only carbohydrate metabolism, but also acylcarnitine profile and selected characteristics of lipid (plasma cholesterol, TAG, NEFA concentrations) and protein metabolism (plasma urea, aspartate aminotransferase (AST), alanine aminotransferase (ALT) and methylmalonylcarnitine concentration) of the true carnivorous cat were scrutinised in the present study. Acylcarnitine profile was studied as a reflection of metabolites available for the citric acid cycle ${ }^{(13)}$. Endocrine characteristics such as leptin and thyroid function were investigated as well, since these characteristics are identified to be related to obesity and insulin resistance. Leptin is distinguished to be strongly and positively correlated with adiposity in obese cats ${ }^{(14)}$ and insulin resistance is associated with increased leptin concentrations in both normal-weight and obese cats ${ }^{(15)}$. Given that thyroid hormones are involved in the regulation of metabolism, and regulate RMR, thermogenesis and lipolysis, thyroid function might be altered by developing obesity ${ }^{(16)}$. Hence, total fasting triiodothyronine $\left(\mathrm{T}_{3}\right)$ and total thyroxine $\left(\mathrm{T}_{4}\right)$ concentrations were also obtained in the present trial.

Seeing that very little is known about the metabolic effects of prebiotics in cats, the purpose of the present trial was to evaluate the effect of adding oligofructose and inulin to a basic diet on feline carbohydrate, lipid and protein metabolism as well as to determine the metabolic differences between healthy normal-weight and obese cats.

\section{Materials and methods}

\section{Animals and housing}

Sixteen domestic short-hair cats, six males and ten females, were included in the study. All male and female cats were neutered. All cats were adult and aged between 3.5 and 6 years. All cats were healthy apart from chronic obesity in eight cats and were not given any medication at the time of the study; none had prior medical problems. During the trial, the cats were housed in individual indoor cages. For $2 \mathrm{~h} / \mathrm{d}$, cats were allowed to play in their usual group cages. At that time, cats had no access to the food, but water was available ad libitum.

\section{Diets and feeding}

The control diet (C-diet) was a non-commercial extruded dry cat food, containing high concentrations of crude protein (46\% on DM basis), moderate amounts of crude fat $(15 \%$ $\mathrm{DM})$ and low concentrations of carbohydrates ( $\mathrm{N}$-free extract $27 \% \mathrm{DM}$ ), in order to trigger the cats towards insulin resistance (A Verbrugghe et al., unpublished results). The food also contained moderate concentrations of crude fibre $(4.6 \%$ DM) and crude ash (6.7\% DM) (Table 1) and was coated with palm oil on the outside of the kibble. No prebiotics and other soluble fibres were added. To make the prebiotic diet (P-diet), 2.5\% mixture of oligofructose and inulin
Table 1. Composition of the control diet (C-diet) and prebiotic diet (P-diet; C-diet $+2.5 \%$ of a mixture of oligofructose and inulin)*

\begin{tabular}{lrr}
\hline Nutrients on DM basis (\%) (analysed) & C-diet & P-diet \\
\hline Crude protein & $46 \cdot 4$ & $44 \cdot 1$ \\
Ether extract & $15 \cdot 2$ & $14 \cdot 9$ \\
Crude ash & $6 \cdot 7$ & $6 \cdot 7$ \\
Crude fibre & $4 \cdot 6$ & $4 \cdot 5$ \\
Starch & $23 \cdot 1$ & $22 \cdot 3$ \\
Sugars & $2 \cdot 4$ & $2 \cdot 4$ \\
N-free extract & $27 \cdot 3$ & $29 \cdot 8$ \\
NSP† & $1 \cdot 8$ & $5 \cdot 2$ \\
Total dietary fibre & $9 \cdot 3$ & $11 \cdot 8$ \\
Metabolisable energy (kJ/100 g as fed) $\ddagger$ & 1453 & 1433 \\
\hline
\end{tabular}

*Ingredients: greaves meal, wheat flour, chicken meal, wheat, bovine chicken fat, linseed, meat and bone meal, brewer's yeast, premium cat digest liquid, fish meal, premix, monosodium glutamate, salt, DLmethionine, iron oxide black, choline chloride $75 \%$.

$\dagger \mathrm{N}$-free extract - starch - sugars.

$\mp$ Calculated: $15 \times$ crude protein $+36 \times$ ether extract $+15 \times$ (starch + sugars).

(Beneo $^{\text {тм }}$ Synergy $1{ }^{\circledR}$; Beneo-Orafti, Beneo-Group, Tienen, Belgium) was added to the $\mathrm{C}$-diet. This soluble fibre mixture is a co-spray dried 1:1 mixture of long-chain chicory inulin molecules, enriched with short-chain oligofructose obtained by partial enzymatic hydrolysis of chicory inulin and containing low concentrations of fructose, glucose and sucrose as well. The mean total number of fructose or glucose units (degree of polymerisation) was 25 for the inulin; mean degree of polymerisation of oligofructose was 4 . The soluble fibre was not mixed with the ingredients, but was added to the palm oil coating. The proximate analysis of the diets is shown in Table 1. Total dietary fibre was determined by acid and enzymatic digestion using enzymes from a commercial test kit (Bioquant Total Dietary Fiber, Merck, USA), followed by correction for protein and ash and is also shown in Table 1 .

The amount of food calculated corresponded to the maintenance energy requirement (normal-weight cats $418 \mathrm{~kJ} /$ $\mathrm{kg}^{0.67}$; obese cats $\left.544 \mathrm{~kJ} / \mathrm{kg}^{0 \cdot 4}\right)^{(17)}$ and was adapted in order to maintain a constant body weight. The food was available all day, except for the $2 \mathrm{~h}$ playtime. Cats were allowed free access to water at all times.

\section{Experimental design}

Prior to being entered into the study, the cats underwent a physical examination, a blood sample was drawn from the jugular vein after a $12 \mathrm{~h}$ fast for complete blood count and serum biochemistry and body weight, body condition score (BCS), BMI and girth circumference were recorded. The BCS was determined using a five-point body condition scoring system $^{(18)}$. Non-obese cats with a score of $3 / 5$ (mean body weight $4.3 \mathrm{~kg}$; range $3.7-5.3 \mathrm{~kg}$ ) and obese cats with a score of $5 / 5$ (mean body weight $6.8 \mathrm{~kg}$; range $5.3-9.8 \mathrm{~kg}$ ) were used in the study. The BMI was calculated as described by Hoenig et al. ${ }^{(19)}$. Girth circumference was measured directly behind the last rib $^{(19)}$. All measurements were performed under general anaesthesia by the same person to minimise variability (Table 2). For 4 weeks preceding the trial (adaptation period), all cats were fed the $\mathrm{C}$-diet prior to being 
Table 2. Body condition score (BCS), body weight, BMI and girth circumference in eight normal-weight and seven obese neutered adult cats

(Mean values and standard deviations)

\begin{tabular}{lrrrrrr}
\hline & \multicolumn{2}{c}{ Normal weight } & & \multicolumn{2}{c}{ Obese } & \\
\cline { 2 - 3 } & Mean & SD & & Mean & SD & \\
\hline BCS & 3.1 & 0.2 & & 5.0 & 0.0 & $<0.001$ \\
Body weight $(\mathrm{kg})$ & 4.3 & 0.6 & & 6.8 & 1.4 & $<0.001$ \\
BMI $\left(\mathrm{kg} / \mathrm{cm}^{2}\right)$ & 37.7 & 3.7 & & 54.4 & 9.2 & $<0.001$ \\
Girth $(\mathrm{cm})$ & 39.6 & 2.5 & & 50.1 & 5.0 & $<0.001$ \\
\hline
\end{tabular}

randomised to one of two groups, each containing four normal-weight and four obese cats. Each group of cats was assigned to each of two diets (C-diet and P-diet) in a random order at intervals of 4 weeks. This way, diets were examined in a crossover design. Absolute food intake was measured daily throughout the study and relative food intake (\% of metabolisable energy intake relative to maintenance energy requirement) was calculated. Body weight was recorded twice weekly.

To determine the effect on glucose and insulin metabolism, an intravenous glucose tolerance test (IVGTT) was performed in each cat at the end of each testing period. Hence, a central venous catheter was placed into a jugular vein to allow glucose administration and blood sampling. At least $20 \mathrm{~h}$ prior to the IVGTT, cats were anaesthetised with buprenorphine $\left(10 \mu \mathrm{g} / \mathrm{kg}\right.$ intravenous; Temgesic ${ }^{\circledR}$; Schering-Plough n.v., Heist-Op-Den-Berg, Belgium), followed by propofol $\left(6-7 \mathrm{mg} / \mathrm{kg}\right.$ to effect, intravenous; Propovet ${ }^{\circledR}$; Abbott Lab, Leuven, Belgium), and a $20 \mathrm{G}, 8 \mathrm{~cm}$ intravenous catheter (Leaderflex ${ }^{\circledR}$, Vygon n.v., Brussels, Belgium) was placed in a jugular vein. Catheters were flushed twice daily with $1 \mathrm{ml}$ heparinised saline (50 IU of heparin/ml in saline $(0.9 \%$ $\mathrm{NaCl})$ solution) to maintain patency. Amoxycilline $(15 \mathrm{mg} / \mathrm{kg}$; Clamoxyl LA ${ }^{\circledR}$; GlaxoSmithKline n.v., Genval, Belgium) was administered subcutaneously once at the time of catheter placement. The IVGTT was performed between 9.00 and 12.00 hours after a $12 \mathrm{~h}$ fast. Glucose (Glucose Sterop $500 \mathrm{mg} / \mathrm{ml}$; Laboratoria Sterop n.v., Brussels, Belgium) was administered $(0.5 \mathrm{~g} / \mathrm{kg})$, through the jugular vein catheter over $30-45 \mathrm{~s}$, followed immediately by $1 \mathrm{ml}$ normal saline to flush the catheter ${ }^{(20)}$. Blood samples were collected from the jugular catheter as described by Martin \& Rand $^{(21)}$, prior to $(0 \mathrm{~min})$ and $2,5,10,15,30,45,60,90$ and $120 \mathrm{~min}$ after glucose administration ${ }^{(20)}$. At time zero, blood samples were collected in tubes containing lithium heparin for determination of AST, ALT, leptin, $\mathrm{T}_{3}$ and $\mathrm{T}_{4}$ concentrations, free carnitine and acylcarnitine profile. Serum tubes were used to determine fasting serum total cholesterol, TAG, NEFA, urea and creatinine concentrations, at time zero. At each time interval, blood samples were collected in tubes containing sodium fluoride for determination of plasma glucose concentrations and in serum tubes for determination of serum insulin concentrations. Plasma and serum were obtained by centrifugation and stored at $-20^{\circ} \mathrm{C}$ until assayed.

The experimental protocol was approved by the Ethical Committee of the Faculty of Veterinary Medicine, Ghent University, Belgium (EC 2007/013).

\section{Analytical methods}

Plasma glucose, serum total cholesterol, TAG, urea and creatinine concentrations were determined spectrophotometrically using the Roche/Hitachi Modular Analyzer (Roche Diagnostics GmbH, Mannheim, Germany). Likewise, activities of AST and ALT were analysed spectrophotometrically using the Roche/Hitachi Modular Analyzer with pyridoxal phosphate activation. Plasma NEFA concentrations were determined enzymatically using a commercially available method (NEFA; Randox Laboratories Ltd, Crumlin, UK) on RX Daytona (Randox). Serum insulin concentrations were measured using a commercially available immunoradiometric assay test kit (insulin immunoradiometric assay Ref 5251; Biosource Europe S.A., Nivelles, Belgium) as used by Slingerland et al. ${ }^{(22)}$. Plasma leptin concentrations were determined using a commercially available RIA test kit (Multi-Species Leptin RIA Kit, catalogue number XL-85K; Linco Research Inc., St. Charles, MO, USA). This kit was developed to quantify leptin in plasma from several species and has been validated for use in cats ${ }^{(23)}$. Fasting plasma $T_{3}$ and $T_{4}$ concentrations were determined using a specific RIA as described by Darras et al. ${ }^{(24)}$. Quantitative electrospray tandem-MS was used for free carnitine and acylcarnitine analysis, as described by Vreken et al. ${ }^{(25)}$ and Rizzo et al. ${ }^{(26)}$.

The glucose disappearance coefficient $\left(K_{\text {gluc }}\right)$ and the halflife for glucose disappearance (glucose $t_{1 / 2}$ ) between 15 and $90 \mathrm{~min}$ after glucose administration were calculated as described by Link \& Rand ${ }^{(27)}$. Area under the glucose curve $\left(\mathrm{AUC}_{\text {gluc }}\right)$ and area under the insulin curve $\left(\mathrm{AUC}_{\mathrm{ins}}\right)$ were calculated according to the trapezoidal method (baseline equal to zero). The basal insulin to glucose ratio and the ratio of area under the insulin to glucose curve $\left(\mathrm{AUC}_{\mathrm{ins}} / \mathrm{AUC}_{\mathrm{gluc}}\right)$ as well as the homeostasis model assessment (HOMA), the quantitative insulin sensitivity check index (QUICKI) and the Bennett index were calculated as described by Appleton et al. ${ }^{(28)}$.

\section{Statistical analysis}

Statistical analysis were performed using Superior Performing Software Systems version 16 (SPSS Inc., Chicago, IL, USA). For body weight, BCS, BMI and girth circumference at the beginning of the trial one-way ANOVA was performed. For plasma glucose and serum insulin concentrations during IVGTT, repeated-measures ANOVA was used, with BCS as between-subject factor and diet and time as within-subject factor. All remaining data, including the different glucose time-points during IVGTT, were statistically analysed by repeated-measures ANOVA with BCS as between-subject factor and diet as within-subject factor. Interactions between BCS and diet were also evaluated, but were not present. Statistical significance was accepted at $P<0.05$. All data are expressed as means and standard deviations.

\section{Results}

All sixteen cats except one completed the trial. One obese cat died as a consequence of an unrelated cause during general anaesthesia. During the first sampling period, the IVGTT 
failed in four cats (one of each group) due to technical problems. In these cats only fasting blood samples could be obtained.

\section{Effect of body condition}

At the start of the trial, body weight, BCS, BMI and girth circumference $(P<0.001$ for all) differed among normal-weight and obese cats (Table 2). During the trial, body weight remained stable in all cats (data not shown). The effect of BCS on feed intake, fasting metabolic and endocrine parameters, regardless of diet, is shown in Table 3 . Obese cats ate more compared with normal-weight cats $(P=0 \cdot 019)$, but no differences were noted in relative food intake. Fasting serum creatinine, NEFA and cholesterol concentrations were comparable between normal-weight and obese cats. However, fasting serum urea concentrations were lower $(P=0.011)$ and serum TAG concentrations tended $(P=0.094)$ to be higher in obese cats when compared to normal-weight cats. Plasma ALT activity was increased in obese cats $(P=0.043)$, but plasma AST activity did not change among obese and normal-weight cats. Fasting plasma leptin and $\mathrm{T}_{3}$ concentrations were also increased in obese cats in contrast to normal-weight cats $(P=0.003$ and $P=0.026$, respectively). Fasting plasma $\mathrm{T}_{4}$ concentrations did not differ in relation to BCS. The effect of BCS on glucose and insulin metabolism, regardless of diet, is shown in Table 4. Obese cats had higher fasting plasma glucose $(P=0 \cdot 030)$, higher glucose concentration at any time during IVGTT, except for 5 and 120 min after glucose administration (also shown in Fig. 1; main effect: $P=0.013$; time $\times$ BCS: $P=0.043$ ), and higher $\operatorname{AUC}_{\text {gluc }}(P=0.022)$. The $K_{\text {gluc }}$ and glucose $t_{1 / 2}$ were not affected by body condition (Table 4$)$. As shown in Fig. 2, body condition did not influence serum insulin concentrations at any time-point, as well as the height of the first and second insulin peak and the appearance time of the first insulin peak. Yet, the second insulin peak was delayed in obese cats when compared to normal-weight cats $(P=0 \cdot 009)$. $\mathrm{AUC}_{\mathrm{ins}}$, fasting basal insulin to glucose ratio and $\mathrm{AUC}_{\mathrm{ins}} / \mathrm{AUC}_{\mathrm{gluc}}$ as well as HOMA, QUICKI and Bennett index were not affected by body condition (Table 4).

\section{Effect of adding $2.5 \%$ mixture of oligofructose and inulin to a control diet}

Both diets were well tolerated. During the trial, none of the cats refused to eat either of the diets and none showed signs of illness or maldigestion. Absolute and relative food intake showed no significant differences between diets. Adding $2.5 \%$ mixture of oligofructose and inulin to the C-diet did not alter characteristics related to glucose and insulin metabolism in healthy normal-weight or obese cats. As shown in Fig. 1, fasting plasma glucose concentration and plasma glucose concentrations at each other time-point after glucose administration were comparable. Also the $\mathrm{AUC}_{\mathrm{gluc}}$, $K_{\text {gluc }}$ and glucose $t_{1 / 2}$ remained unaffected. Similarly, fasting insulin, $\mathrm{AUC}_{\mathrm{ins}}$ as well as height and appearance time of the insulin peaks were similar with both diets with slightly, though not significantly, higher release with fructan intervention (Fig. 2). All endocrine (leptin, $\mathrm{T}_{3}, \mathrm{~T}_{4}$ ) and metabolic (cholesterol, NEFA, urea, creatinine, ALT) characteristics remained stable, except for serum TAG, which tended to be increased $(P=0.065)$ and plasma AST activity which was decreased $(P=0.025)$ when fed the P-diet compared to the C-diet. As shown in Table 5, plasma free carnitine concentrations did not differ among diets, as did plasma acetylcarnitine concentrations. Yet, propionylcarnitine $(P=0.03)$ and butyrylcarnitine $(P=0.002)$ were higher when fed the P-diet. Methylmalonylcarnitine tended to be decreased $(P=0.072)$ when fed the P-diet.

Table 3. Effect of body condition score on feed intake, fasting metabolic and endocrinologic characteristics, regardless of diet ${ }^{\star}$

(Mean values and standard deviations)

\begin{tabular}{|c|c|c|c|c|c|c|c|}
\hline & \multicolumn{6}{|c|}{ Body condition } & \multirow[b]{3}{*}{$P$} \\
\hline & \multicolumn{3}{|c|}{ Normal weight } & \multicolumn{3}{|c|}{ Obese } & \\
\hline & Mean & SD & $n \dagger$ & Mean & SD & $n \dagger$ & \\
\hline Absolute food intake $(\mathrm{g} / \mathrm{d})$ & $48 \cdot 0^{a}$ & $7 \cdot 9$ & 16 & $61 \cdot 9^{b}$ & $11 \cdot 4$ & 14 & 0.019 \\
\hline Relative food intake (\%) & 94.6 & $7 \cdot 2$ & 16 & 93.9 & $4 \cdot 0$ & 14 & 0.822 \\
\hline Urea $(\mathrm{mmol} / \mathrm{l})$ & $9 \cdot 2^{\mathrm{a}}$ & $2 \cdot 1$ & 16 & $6 \cdot 7^{\mathrm{b}}$ & $1 \cdot 3$ & 14 & 0.011 \\
\hline Creatinine $(\mu \mathrm{mol} / \mathrm{l})$ & 131 & 26 & 16 & 117 & 21 & 14 & 0.252 \\
\hline AST (U/I) & $36 \cdot 0$ & $24 \cdot 1$ & 16 & $28 \cdot 8$ & $11 \cdot 8$ & 14 & 0.309 \\
\hline ALT (U/l) & $28 \cdot 8^{\mathrm{a}}$ & $13 \cdot 0$ & 16 & $49 \cdot 7^{b}$ & 34.9 & 14 & 0.043 \\
\hline Cholesterol (mmol/l) & $3 \cdot 1$ & 0.6 & 16 & $3 \cdot 3$ & 0.9 & 14 & 0.574 \\
\hline $\mathrm{TAG}(\mu \mathrm{mol} / \mathrm{l})$ & 0.42 & 0.2 & 15 & 0.51 & 0.4 & 12 & 0.094 \\
\hline NEFA (mmol/l) & $1 \cdot 24$ & 0.4 & 15 & $1 \cdot 28$ & 0.6 & 12 & 0.783 \\
\hline Leptin (ng/ml) & $7 \cdot 4^{\mathrm{a}}$ & $2 \cdot 8$ & 16 & $20 \cdot 2^{b}$ & $11 \cdot 2$ & 12 & 0.003 \\
\hline $\mathrm{T}_{3}(\mathrm{ng} / \mathrm{ml})$ & $0.26^{a}$ & 0.08 & 16 & $0.34^{b}$ & 0.06 & 13 & 0.026 \\
\hline $\mathrm{T}_{4}(\mathrm{ng} / \mathrm{ml})$ & 4.4 & 0.8 & 16 & $5 \cdot 1$ & $2 \cdot 7$ & 14 & 0.472 \\
\hline
\end{tabular}

ALT, alanine aminotransferase; AST, aspartate aminotransferase; $T_{3}$, tri-iodothyronine; $T_{4}$, thyroxine

a,b Mean values within a row with unlike superscript letters were significantly different $(P<0.05)$.

* For details of procedures, see Materials and methods.

$\dagger n<16$ caused by missing values. 
Table 4. Effect of body condition score on glucose and insulin metabolism, regardless of diet* (Mean values and standard deviations)

\begin{tabular}{|c|c|c|c|c|c|c|c|}
\hline & \multicolumn{6}{|c|}{ Body condition } & \multirow[b]{3}{*}{$P$} \\
\hline & \multicolumn{3}{|c|}{ Normal weight } & \multicolumn{3}{|c|}{ Obese } & \\
\hline & Mean & SD & $n \dagger$ & Mean & SD & $n \dagger$ & \\
\hline Glucose nadir $(\mathrm{mmol} / \mathrm{l})$ & $4.5^{\mathrm{a}}$ & 0.5 & 16 & $5 \cdot 1^{\mathrm{b}}$ & 0.7 & 14 & 0.030 \\
\hline $\mathrm{AUC}_{\text {gluc }}(\mathrm{mmol} / \mathrm{l}$ per $120 \mathrm{~min})$ & $1247^{a}$ & 214 & 14 & $1533^{b}$ & 170 & 12 & 0.022 \\
\hline$K_{\text {gluc }}$ & 1.24 & 0.34 & 14 & 1.22 & 0.26 & 12 & 0.967 \\
\hline Glucose $t_{1 / 2}(\mathrm{~min})$ & $61 \cdot 3$ & 23.5 & 14 & 59.5 & 14.9 & 12 & 0.798 \\
\hline Insulin nadir (mU/l) & $16 \cdot 5$ & $5 \cdot 7$ & 16 & $17 \cdot 2$ & $7 \cdot 0$ & 14 & 0.820 \\
\hline$A U C_{\text {ins }}(\mathrm{mU} / \mathrm{l}$ per $120 \mathrm{~min})$ & 4467 & 2083 & 14 & 5186 & 1640 & 12 & 0.745 \\
\hline Height 1 st insulin peak (mU/l) & 35.4 & $14 \cdot 1$ & 14 & 30.5 & $14 \cdot 0$ & 12 & 0.239 \\
\hline Height 2nd insulin peak (mU/l) & $57 \cdot 6$ & $27 \cdot 9$ & 14 & $65 \cdot 8$ & $25 \cdot 5$ & 12 & 0.777 \\
\hline Time 1st insulin peak (min) & 8.9 & 4.8 & 14 & 11.3 & $3 \cdot 8$ & 12 & 0.430 \\
\hline Time 2nd insulin peak (min) & $57 \cdot 9^{\mathrm{a}}$ & $15 \cdot 4$ & 14 & $78 \cdot 8^{\mathrm{b}}$ & $21 \cdot 3$ & 12 & 0.009 \\
\hline Fasting $\mathrm{Sl}_{1}(\mathrm{mU} / \mathrm{mmol})$ & $3 \cdot 7$ & $1 \cdot 2$ & 16 & 3.3 & $1 \cdot 1$ & 12 & 0.469 \\
\hline$A \cup C_{\text {ins }} / A \cup C_{\text {gluc }}(\mathrm{mU} / \mathrm{mmol})$ & 3.6 & 1.6 & 14 & 3.4 & $1 \cdot 1$ & 14 & 0.683 \\
\hline HOMA & 3.3 & 1.3 & 16 & $4 \cdot 1$ & $2 \cdot 0$ & 14 & 0.380 \\
\hline QUICKI & 0.56 & 0.09 & 16 & 0.53 & 0.07 & 14 & 0.481 \\
\hline Bennett index $\neq$ & 1.4 & 0.4 & 16 & $1 \cdot 2$ & 0.3 & 14 & 0.370 \\
\hline \multicolumn{8}{|c|}{$\begin{array}{l}\mathrm{AUC}_{\text {gluc, }} \text { area under the curve for glucose; } \mathrm{AUC}_{\text {ins }} \text {, area under the curve for insulin; fasting } \mathrm{Sl}_{1} \text {, basal insulin to glucose ratio; } \\
\text { glucose } t_{1 / 2} \text {, half-life for glucose disappearance between } 15 \text { and } 90 \text { min after glucose administration; HOMA, homeostasis } \\
\text { model assessment; } K_{\text {gluc }} \text {, glucose disappearance coefficient; QUICKI, quantitative insulin sensitivity check index. } \\
\text { a,b Mean values within a row with unlike superscript letters were significantly different }(P<0.05) \text {. } \\
{ }^{*} \text { For details of procedures, see Materials and methods. }\end{array}$} \\
\hline
\end{tabular}

\section{Discussion}

In the current study, obese cats confirmed a higher risk for insulin resistance and impaired glucose tolerance than normal-weight cats. Under the test conditions, differences in main blood glucose control characteristics were not observed. Obese cats, taking part in the present trial, had no significantly higher fasting serum insulin concentrations, but the increased $\mathrm{AUC}_{\text {gluc }}$, the higher fasting plasma glucose concentration and the later appearance of the second insulin peak during IVGTT may have resulted in an impaired glucose tolerance and a higher insulin resistance in obese cats regardless of diet. The present findings were also concluded from previous studies in cats ${ }^{(29,30)}$. The significantly higher fasting plasma leptin concentrations in obese cats might also predict the occurrence of insulin resistance, since leptin is postulated to mediate some of the metabolic consequences of obesity. In cats, Appleton et al. ${ }^{(15)}$ have demonstrated a strong positively relationship between leptin and insulin resistance. The significantly higher fasting plasma $T_{3}$ concentrations in obese cats during the present trial corresponds to the significant and positive correlation of $\mathrm{T}_{3}$ with body weight, girth circumference and BMI as observed by Ferguson et al. ${ }^{(16)}$. $\mathrm{T}_{4}$ also correlated positively with all indices of obesity and with leptin, but not with $\mathrm{NEFA}^{(16)}$. Still, fasting $\mathrm{T}_{4}$ concentrations remained unchanged in the present trial. Ferguson et al. ${ }^{(16)}$ also

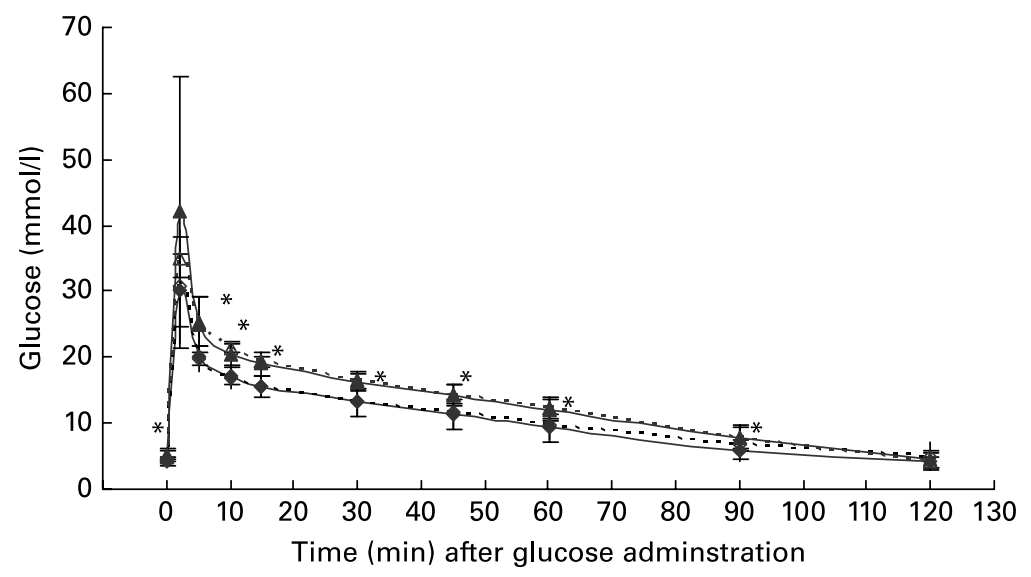

Fig. 1. Plasma glucose concentrations during the intravenous glucose tolerance test in healthy normal-weight (body condition score (BCS) $3 / 5$ ) and obese (BCS 5/5) cats fed the control diet (C-diet) and prebiotic diet (P-diet; C-diet $+2.5 \%$ of a mixture of oligofructose and inulin). $\bullet$, BCS $3, \mathrm{C}$-diet; $\diamond, \mathrm{BCS} 3, \mathrm{P}$-diet; $\triangle$, BCS 5, C-diet; $\triangle$, BCS 5, P-diet. Values are means with their standard deviations depicted by vertical bars. Mean values were significantly different at each different time-point between normal-weight and obese cats, regardless of dietary treatment: ${ }^{*} P<0.05$. 


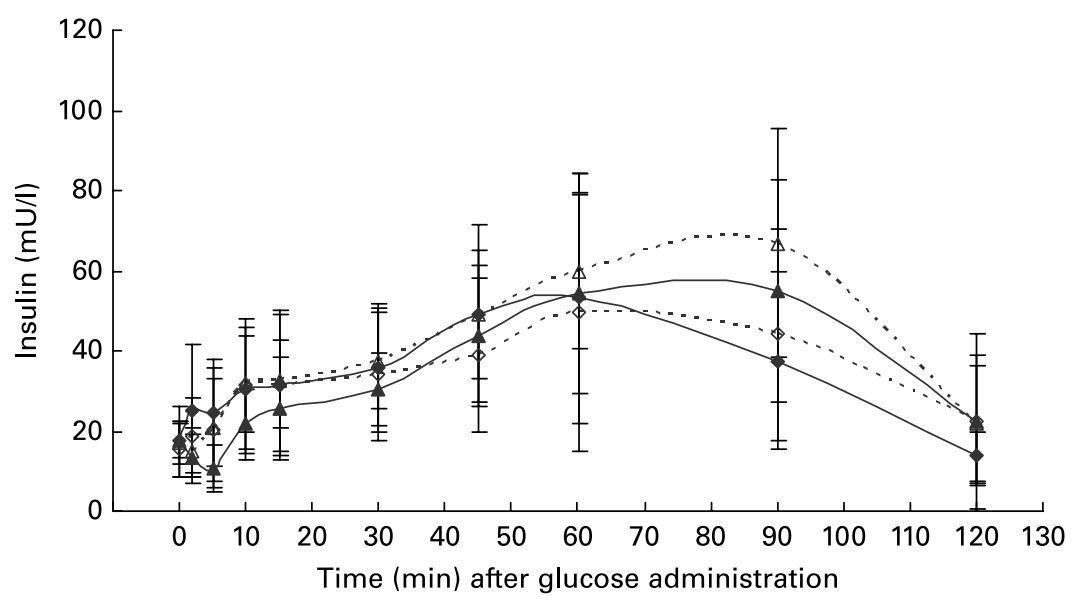

Fig. 2. Serum insulin concentrations during the intravenous glucose tolerance test in healthy normal-weight (BCS $3 / 5)$ and obese (BCS 5/5) cats fed the control diet (C-diet) and prebiotic diet (P-diet; C-diet $+2.5 \%$ of a mixture of oligofructose and inulin). $\bullet$, BCS 3 , C-diet; $\diamond$, BCS 3, P-diet; $\mathbf{\Lambda}$, BCS 5, C-diet; $\triangle$, BCS 5 , P-diet. Values are means with their standard deviations depicted by vertical bars.

demonstrated increased leptin and NEFA after weight gain and proposed an obesity-induced relative state of thyroid hormone resistance, either caused by leptin or by increased NEFA concentrations, or both. In the present trial, fasting leptin concentrations were indeed elevated in obese cats, but no significant differences were noted for serum NEFA concentrations. This supports that the relative state of thyroid hormone resistance might be most probably explained by the rise in fasting plasma leptin concentrations. In overweight man, hyperleptinaemia was also observed to be strongly correlated with elevated serum ALT activity ${ }^{(31)}$, most probably due to the high association between elevated liver enzymes, most notably high serum ALT activity and obesity (percentage body fat $^{(32)}$; obesity class ${ }^{(33)}$ ) and insulin resistance (measured by hyperinsulinaemic euglycaemic clamp technique ${ }^{(32)}$ or HOMA $^{(33)}$ ) as observed by Vozarova et al. ${ }^{(32)}$ and Marchesini et al. ${ }^{(33)}$. Therefore, the occurrence of insulin resistance might explain the rise in plasma ALT activity observed in obese cats. In addition, serum TAG also tended to rise in obese cats, in accordance with the results found by Hoenig et al. ${ }^{(19)}$, who studied the effect of obesity on lipid profiles in neutered cats. Hoenig et al. ${ }^{(19)}$ also noted a significant rise in plasma total cholesterol concentrations in obese cats; yet, this could not be demonstrated from the present trial. At last, fasting serum urea concentrations were reduced in obese cats compared to normal-weight cats. In rats made obese by feeding a cafeteria-diet, similar results were demonstrated. According to Barber et $_{\text {al. }}{ }^{(34)}$, a decreased serum urea concentration in obese rats might be due to a decrease in the activities of all enzymes of the urea cycle and a lower rate of synthesis of urea from precursors in hepatocytes. To date, no data are available on the impact of feline obesity on urea concentrations and the activity of urea cycle enzymes, yet in obese cats suffering from severe hepatic lipidosis it is observed that blood urea nitrogen might be subnormal caused by chronic anorexia or presuming impaired urea cycle function ${ }^{(35)}$. However, during the present trial cats were not anorectic, did not lose any weight and did not show any other clinical signs reflecting hepatic lipidosis, which probably indicates reduced serum urea concentrations due to compromised urea cycle function as observed in obese rats.

Table 5. Effect of adding $2.5 \%$ of a mixture of oligofructose and inulin to the control diet on carnitine metabolism, regardless of body condition*

(Mean values and standard deviations)

\begin{tabular}{|c|c|c|c|c|c|c|c|}
\hline & \multicolumn{6}{|c|}{ Diet } & \multirow[b]{3}{*}{$P$} \\
\hline & \multicolumn{3}{|c|}{ C-diet } & \multicolumn{3}{|c|}{ P-diet } & \\
\hline & Mean & SD & $n \dagger$ & Mean & SD & $n \dagger$ & \\
\hline Free carnitine & $32 \cdot 15$ & 6.07 & 15 & 41.42 & 21.03 & 15 & 0.579 \\
\hline Acetylcarnitine & 5.545 & 0.823 & 15 & 6.321 & 2.500 & 15 & 0.352 \\
\hline Propionylcarnitine & $0.134^{\mathrm{a}}$ & 0.082 & 15 & $0.275^{\mathrm{b}}$ & 0.109 & 15 & 0.030 \\
\hline Butyrylcarnitine & $0.283^{a}$ & 0.069 & 15 & $0.474^{\mathrm{b}}$ & 0.186 & 15 & 0.002 \\
\hline Methylmalonylcarnitine & 0.054 & 0.029 & 15 & 0.036 & 0.012 & 15 & 0.072 \\
\hline
\end{tabular}

C-diet, control diet; P-diet, prebiotic diet (C-diet $+2.5 \%$ of a mixture of oligofructose and inulin).

a,b Mean values within a row with unlike superscript letters were significantly different $(P<0.05)$.

${ }^{*}$ For details of procedures and diets, see the Materials and methods section and Table 1.

$\dagger n<16$ caused by missing values. 
Adding $2.5 \%$ mixture of oligofructose and inulin to a basic diet did not alter the investigated standard characteristics for glucose and insulin metabolism, or other metabolic and endocrine characteristics, in healthy normal-weight and obese cats; except for serum TAG concentrations, the activity of AST and several acylcarnitines. Serum TAG concentrations tended to be increased in cats supplemented with oligofructose and inulin. The present finding contrasts with the results from earlier trials in rodents ${ }^{(36-40)}$ and $\operatorname{dogs}{ }^{(8)}$ which revealed lower TAG concentrations following soluble fibre supplementation. Since body weight remained unchanged during the trial, an increase in body weight could not explain the present observation. Nevertheless, serum TAG concentrations remained within the references range. When compared to other trials conducted in rats ${ }^{(39-41)}$ and $\operatorname{dogs}^{(8-10,12)}$, the amount of soluble fibre used in the present trial $(0.5 \mathrm{~g} / \mathrm{kg}$ body weight $)$ seems rather low. Nevertheless, in human studies ${ }^{(42-44)}$ doses of oligofructose were similar to the dose used in the present trial. In healthy human subjects ingesting $20 \mathrm{~g}$ fructooligosaccharide/d $(0 \cdot 3 \mathrm{~g} / \mathrm{kg}$ body weight $)$ for 4 weeks, fructooligosaccharides did not modify fasting glucose and insulin concentrations, but did lower basal hepatic glucose production $^{(42)}$. According to Yamashita et al. ${ }^{(45)}, 8 \mathrm{~g}$ fructooligosaccharide/d $(0 \cdot 1 \mathrm{~g} / \mathrm{kg}$ body weight $)$ for 2 weeks resulted in a reduced fasting glycaemia in type 2 diabetic patients. For the results of the current study, species differences including insulin sensitivity must be taken into account. For higher doses, Hesta et al. ${ }^{(46)}$ showed that faeces became formless and apparent digestibility of protein and fat were reduced when cats ingested more then $3 \%$ oligofructose or inulin, suggesting a limitation of more than $3 \%$ oligofructose or inulin for practical use in cats. Concerning the type of soluble fibre, Diez et al. ${ }^{(8)}$ observed significantly decreased postprandial glucose concentrations after incorporation of both fructooligosaccharides and sugar beet fibre in healthy dogs, in spite of non-significant effects on fasting glucose concentration or postprandial glucose curve after supplementation of inulin or sugar-beet fibre ${ }^{(9)}$. In the present trial the diet was supplemented with a mixture of both oligofructose and inulin, containing a higher proportion of inulin which has a higher degree of polymerisation compared to oligofructose. This suggests a slower fermentation in the relative short large bowel of the cat. Another reason for the absence of effect on standard characteristics for glucose and insulin metabolism might be the control diet. At first, it has been demonstrated in $\operatorname{man}^{(47)}$ and $\operatorname{dogs}^{(48)}$ that supplementing soluble as well as insoluble fibre to a basic diet only results in a better glucose and insulin response when the diet contains more than $40 \%$ of the metabolisable energy as carbohydrates, whereas our basic diet only contained $26 \%$ of metabolisable energy as carbohydrates. Secondly, insulin resistance might have been triggered better by using a diet containing high amounts of fat, as demonstrated in man and rodents ${ }^{(49,50)}$ as well as in cats ${ }^{(51)}$, instead of a high-protein, low-carbohydrate diet as used in the present trial. Nevertheless, a previous trial showed an increased insulin resistance in healthy non-obese cats fed a low-carbohydrate diet when compared to a lowfat and a low-protein diet (A Verbrugghe et al., unpublished results). Mechanisms responsible for enhancing glucose tolerance and insulin sensitivity due to soluble fibre as described in other species might be less pronounced in cats.
Despite the absence of a direct effect on glucose tolerance and insulin sensitivity, an effect on colonic fermentation and the production of SCFA could be demonstrated by acylcarnitine analysis. Higher production of propionate and butyrate and metabolisation of these SCFA were observed by the higher propionyl- and butyrylcarnitine concentrations in cats when fed the P-diet. Acetylcarnitine was not altered among diets. Acetyl-CoA is not only generated from acetate, but also from amino acids and other fatty acids. Moreover, propionate might inhibit the rise in acetylcarnitine concentrations caused by butyrate, as demonstrated by Brass \& Beyerinck ${ }^{(52)}$.

In the present trial, oligofructose and inulin might have contributed to the citric acid cycle through propionate. Theoretically, propionate, being a gluconeogenic SCFA, can be converted to glucose via succinyl-CoA and oxaloacetate ${ }^{(53)}$. In ruminants ${ }^{(54,55)}$ and horses ${ }^{(56)}$, propionate is known to be the primary gluconeogenic substrate. In strictly carnivorous species, such as cats, no data are available yet. It is also known from studies in man as well as experimental animals that propionate decreases gluconeogenesis from pyruvate. This occurs directly through inhibition of pyruvate carboxylase via its specific intermediaries, methylmalonyl CoA and propionyl CoA and indirectly through depletion of acetyl CoA, a specific allosteric activator of this enzyme ${ }^{(57,58)}$. The present trial revealed increased propionylcarnitine concentrations, suggesting inhibited gluconeogenesis from pyruvate, resulting in sparing amino acids. Methylmalonylcarnitine tended to decrease among diets, which supports the hypothesis of reduced amino acid catabolism, since methylmalonylcarnitine is known to be a metabolite of valine, methionine and isoleucine catabolism. Moreover, the significantly decreased plasma AST activity in cats supplemented with oligofructose and inulin indicates inhibited gluconeogenesis from aspartate.

In conclusion, impaired glucose tolerance and increased insulin resistance were observed in obese cats compared to normal-weight cats, regardless of diet. Adding $2.5 \%$ of a mixture of oligofructose and inulin to a basic diet did not affect glucose tolerance in healthy normal-weight or in obese cats. However, modulation of glucose metabolism by enhancing gluconeogenesis from propionate and therefore inhibition of amino acid catabolism can be suggested, and can be beneficial in the treatment of feline insulin resistance and diabetes. Yet, further research investigating the postprandial effects of prebiotics on acylcarnitine profile can be of interest.

\section{Acknowledgements}

This study is within the scope of the postgraduate study of A.V. and is funded by the Institute for Promotion of Innovation through Science and Technology in Flanders (IWT-Vlaanderen). Expenses for the laboratory analyses were covered by the Beneo-Orafti, Beneo-Group (Tienen, Belgium), who also provided the oligofructose and inulin mixture $\left(\right.$ Beneo ${ }^{\mathrm{TM}}$ Synergy $1^{\circledR}$ ). The pet food was provided by Versele-Laga (Deinze, Belgium). A. V. was responsible for the study design, study performance, characteristic analysis, data analysis and manuscript drafting. K. G. was responsible for catheter placement and contributed to manuscript drafting. B. W. and J. B. supervised the plasma analyses and also contributed to the manuscript drafting. M. H., S. D. and G. P. J. J., 
all three promoters of $\mathrm{A}$. V. contributed to the development of the study design, data analysis and manuscript drafting. At last, we gratefully acknowledge Herman De Rycke for performing the food analyses, Inge Vaesen for the plasma analyses, Rebekka Hollebosch and Steven Galle for taking care of the experimental animals, and Stephanie Van Weyenberg and Georgios Papadopoulos for technical assistance. It is declared by the corresponding author that no conflict of interest exists for this paper.

\section{References}

1. Prahl A, Guptill L, Glickman NW, et al. (2007) Time trends and risk factors for diabetes mellitus in cats presented to veterinary hospitals. J Feline Med Surg 9, 351-358.

2. Rand JS, Fleeman LM, Farrow HA, et al. (2004) Canine and feline diabetes mellitus: nature or nurture? J Nutr 134, 2072S-2080S.

3. Roberfroid MB \& Delzenne NM (1998) Dietary fructans. Annu Rev Nutr 18, 117-143.

4. Blaxter AC, Cripps PJ \& Gruffydd-Jones TJ (1990) Dietary fibre and postprandial hyperglycemia in normal and diabetic dogs. J Small Anim Pract 31, 229-233.

5. Nelson RW (1989) The role of fibre in managing diabetes mellitus. Vet Med 84, 1156-1160.

6. Nelson RW (1992) Dietary management of diabetes mellitus. J Small Anim Pract 33, 213-217.

7. Delarue J \& Magnan C (2007) Free fatty acids and insulin resistance. Curr Opin Clin Nutr Metab Care 10, 142-148.

8. Diez M, Hornick J-L, Baldwin P, et al. (1997) Influence of a blend of fructo-oligosaccharides and sugar beet fibre on nutrients digestibility and plasma metabolite concentrations in healthy beagle dogs. Am J Vet Res 58, 1238-1242.

9. Diez M, Hornick J-L, Baldwin P, et al. (1998) Influence of sugar-beet fibre, guar gum and inulin on nutrient digestibility, water consumption and plasma metabolites in healthy beagle dogs. Res Vet Sci 64, 91-96.

10. Massimino SP, McBurney MI, Field CJ, et al. (1998) Fermentable dietary fibre increases GLP-1 secretion and improves glucose homeostasis despite increased intestinal glucose transport capacity in healthy dogs. J Nutr 128, 1786-1793.

11. Stirckling J, Harmon D, Gross K, et al. (2000) Evaluation of oligosaccharide addition to dog diets: influences on nutrient digestion and microbial populations. Anim Feed Technol 86, 205-219.

12. Hesta M, Debraekeleer J, Janssens GPJ, et al. (2001) The effect of a commercial high fibre diet and an iso-malto-oligosaccharide supplemented diet on postprandial glucose concentrations in dogs. J Anim Physiol Anim Nutr 85, 217-221.

13. Bremer J (1983) Carnitine - metabolism and functions. Physiol Rev 63, 1420-1480.

14. Appleton DJ, Rand JS \& Sunvold GD (2000) Plasma leptin concentrations in cats: reference range, effects of weight gain and relationship with adiposity as measured by dual energy X-ray absorptiometry. J Feline Med Surg 2, 191-199.

15. Appleton DJ, Rand JS \& Sunvold GD (2002) Plasma leptin concentrations are independently associated with insulin resistance in lean and overweight cats. J Feline Med Surg 4, 83-93.

16. Ferguson DC, Caffall Z \& Hoenig M (2007) Obesity increases free thyroxine proportionally to nonesterified fatty acid concentrations in adult neutered female cats. $J$ Endocrinol 194, 267-273.

17. National Research Council (2006) Energy. In Nutrient Requirements of Dogs and Cats, pp. 28-48. Washington, DC: The National Academies Press.
18. Scarlet JM, Donoghue S, Saidla J, et al. (1994) Overweight cats: prevalence and risk factors. Int J Obes 18, Suppl. 1, S22-S28.

19. Hoenig M, Wilkins C, Holson JC, et al. (2003) Effects of obesity on lipid profiles in neutered male and female cats. $\mathrm{Am}$ $J$ Vet Res 64, 299-303.

20. Appleton DJ, Rand JS, Priest J, et al. (2001) Determination of reference values for glucose tolerance, insulin tolerance, and insulin sensitivity tests in clinically normal cats. Am J Vet Res 62, 630-636.

21. Martin GJW \& Rand JS (1999) Evaluation of a polyurethane jugular catheter in cats placed using a modified Seldinger technique. Aust Vet J 77, 250-254.

22. Slingerland LI, Robben JH, van Haeften TW, et al. (2007) Insulin sensitivity and $\beta$-cell function in healthy cats: assessment with the use of the hyperglycaemic glucose clamp. Horm Metab Res 39, 341-346.

23. Backus RC, Havel PJ, Gingerich RL, et al. (2000) Relationship between serum leptin immunoreactivity and body fat mass as estimated by use of a novel gas-phase Fourier transformer infrared spectroscopy deuterium dilution method in cats. Am J Vet Res 61, 796-801.

24. Darras VM, Visser TJ, Berghman LR, et al. (1992) Ontogeny of type I and type III deiodinase activities in embryonic and posthatch chickens: relationship with changes in plasma triiodothyronine and growth hormone levels. Comp Biochem Physiol A Mol Integr Physiol 103, 131-136.

25. Vreken P, van Lint AE, Bootsma AH, et al. (1999) Rapid diagnosis of organic acidemias and fatty-acid oxidation defects by quantitative electrospray tandem-MS acyl-carnitine analysis in plasma. Adv Exp Med Biol 466, 327-337.

26. Rizzo C, Boenzi S, Wanders RJA, et al. (2003) Characteristic acyl-carnitine profiles in inherited defects of peroxisome biogenesis: a novel tool for screening diagnosis using tandem mass spectrometry. Pediatr Res 53, 1013-1018.

27. Link RJ \& Rand JS (1998) Reference values for glucose tolerance and glucose tolerance status in cats. J Am Vet Med Assoc 213, 492-496.

28. Appleton DJ, Rand JS \& Sunvold GD (2005) Basal plasma insulin and homeostasis model assessment (HOMA) are indicators of insulin sensitivity in cats. J Feline Med Surg 7, $183-193$

29. Nelson RW, Himsel CA, Feldman EC, et al. (1990) Glucose tolerance and insulin response in normal weight and obese cats. Am J Vet Res 51, 1357-1362.

30. Hoenig M, Thomaseth K, Brandao J, et al. (2006) Assessment and mathematical modelling of glucose turnover and insulin sensitivity in lean and obese cats. Domest Anim Endocrinol 31, 373-389.

31. Ruhl CE \& Everhart JE (2003) Determinants of the association of overweight with elevated serum alanine aminotransferase activity in the United States. Gastroenterology 124, 71-79.

32. Vozarova B, Stefan N, Lindsay RS, et al. (2002) High alkaline aminotransferase is associated with decreased hepatic insulin sensitivity and predicts the development of type 2 diabetes. Diabetes 51, 1889-1895.

33. Marchesini G, Avagnina S, Barantani EG, et al. (2005) Aminotransferase and gamma-glutamyltranspeptidase levels in obesity are associated with insulin resistance and the metabolic syndrome. J Endocrinol Invest 28, 333-339.

34. Barber T, Vina JR, Vina J, et al. (1985) Decreased urea synthesis in cafeteria-diet-induced obesity in the rat. Biochem $J$ 230, 675-681.

35. Center SA (1993) A retrospective study of 77 cats with severe hepatic lipidosis: 1975-1990. J Vet Int Med 7, 349-359.

36. Levrat M-A, Rémésy C \& Demigné C (1991) High propionate fermentations and mineral accumulation in the caecum of rats adapted to different levels of inulin. J Nutr 121, 1730-1737. 
37. Delzenne NM, Kok N, Fiordaliso M-F, et al. (1993) Dietary fructooligosaccharides modify lipid metabolism in rats. Am J Clin Nutr 57, 820S

38. Fiordaliso M, Kok N, Desager J-P, et al. (1995) Dietary oligofructose lowers triglycerides, phospholipids and cholesterol in serum and very low density lipoproteins of rats. Lipids 30, $163-167$.

39. Agheli N, Kabir M, Berni-Canani S, et al. (1998) Plasma lipids and fatty acid synthase activity are regulated by short chain fructo-oligosaccharides in sucrose-fed insulin-resistant rats. J Nutr 128, 1283-1288.

40. Busserolles J, Gueux E, Rock E, et al. (2003) Oligofructose protects against hypertriglyceridemic and pro-oxidative effect of a high fructose diet in rats. J Nutr 133, 1903-1908.

41. Kok N, Roberfroid M, Robert A, et al. (1996) Involvement of lipogenesis in the lower VLDL secretion induced by oligofructose in rats. Br J Nutr 76, 881-890.

42. Luo J, Rizkalla SW, Alamowitch C, et al. (1996) Chronic consumption of short-chain fructooligosaccharides by healthy subjects decreased basal hepatic glucose production but had no effect on insulin-stimulated glucose metabolism. Am J Clin Nutr 63, 939-945.

43. Alles MS, de Roos NM, Bakx JC, et al. (1999) Consumption of fructooligosaccharides does not favourably affect blood glucose and serum lipid concentrations in patients with type 2 diabetes Am J Clin Nutr 69, 64-69.

44. Luo J, Van Yperselle M, Rizkalla SW, et al. (2000) Chronic consumption of short-chain fructo-oligosaccharides does not affect basal hepatic glucose production or insulin resistance in type II diabetes. J Nutr 130, 1572-1577.

45. Yamashita K, Kawai K \& Itakura K (1984) Effect of fructooligosaccharides on blood glucose and serum lipids in diabetic subjects. Nutr Res 4, 961-966.

46. Hesta M, Janssens GPJ, Debraekeleer J, et al. (2001) The effect of oligofructose and inulin on faecal characteristics and nutrient digestibility in healthy cats. J Anim Physiol Anim Nutr 85, 135-141.

47. Jenkins DJA, Wolever TMS, Bacon S, et al. (1980) Diabetic diets: high carbohydrate combined with high fiber. Am J Clin Nutr 33, 1729-1733.

48. Nelson RW, Ihle SL, Lewis LD, et al. (1991) Effects of dietary fiber supplementation on glycemic control in dogs with alloxaninduced diabetes mellitus. Am J Vet Res 52, 2060-2066.

49. Storlien LH, Baur LA, Kriketos AD, et al. (1996) Dietary fats and insulin action. Diabetologia 39, 621-631.

50. Lichtenstein AH \& Swab US (2000) Relationship of dietary fat to glucose metabolism. Atherosclerosis 150, 227-243.

51. Thiess S, Becskei C, Tomsa K, et al. (2004) Effects of high carbohydrate and high fat diet on plasma metabolite levels and on iv glucose tolerance test in intact and neutered male cats. J Feline Med Surg 6, 207-218.

52. Brass EP \& Beyerinck RA (1988) Effects of propionate and carnitine on the hepatic oxidation of short- and medium-chain length fatty acids. Biochem $J \mathbf{2 5 0}, 819-825$.

53. Wolever TMS, Brighenti F, Royall D, et al. (1989) Effect of rectal infusion of short chain fatty acids in human subjects. Am J Gastroenterol 84, 1027-1033.

54. Judson GJ, Anderson E, Luick JR, et al. (1968) The contribution of propionate to glucose synthesis in sheep given diets of different grain content. Br J Nutr 22, 69-75.

55. Yost WM, Young JW, Schmidt SP, et al. (1977) Gluconeogenesis in ruminants: propionic acid production from a high-grain diet fed to cattle. J Nutr 107, 2036-2043.

56. Simmons HA \& Ford EJ (1991) Gluconeogenesis from propionate produced in the colon of the horse. Br Vet $J 147,340-345$.

57. Blair JB, Cook DE \& Lardy HA (1973) Interaction of propionate and lactate in the perfused rat liver. J Biol Chem 248, 3608-3614.

58. Anderson JW \& Bridges SR (1984) Short chain fatty acid fermentation products of plant fiber affect glucose metabolism of isolated rat hepatocytes. Proc Soc Exp Biol Med 177, 372-376. 